\section{La relación entre obesidad y complicaciones en el curso clínico de las enfermedades respiratorias virales en niños ¿un nuevo factor de riesgo a considerar?}

\author{
LORETO FUENZALIDA ${ }^{1, a, b}$, DIEGO F. GARCÍA-DÍAZ
}

'Instituto de Ciencias Biomédicas, Universidad Autónoma de Chile, Santiago, Chile. ${ }^{2}$ Departamento de Nutrición, Facultad de Medicina, Universidad de Chile, Santiago, Chile.

angeniero en Biotecnología Molecular.

${ }^{\mathrm{b} P h D}$.

Proyecto Fondecyt Iniciación 11150599.

Recibido el 12 de septiembre de 2015, aceptado el 9 de marzo de 2016. during acute respiratory infections in children

Obesity has a high prevalence among children. On the other hand, acute respiratory infections especially of viral origin, are an important cause of morbidity and mortality in this age group. During the recent pandemic of influenza A (H1N1) virus, obesity was identified as a novel independent risk factor for severity multiple markers of the disease. We reviewed the evidence associating obesity with a worse course of respiratory diseases in children. Nine out of 40 retrieved articles, were chosen to be reviewed. We concluded that there is evidence suggesting that immunomodulatory effects of obesity could be considered as a novel risk factor. Thus, bearing in mind the drastic rise in obesity prevalence around the world and in Chile, and the latent possibility of new respiratory pandemics caused by viruses, studying the possible effect of obesity aggravating viral respiratory infections will become important.

(Rev Med Chile 2016; 144: 1177-1184)

Key words: Inflammation; Obesity; Pediatric Obesity; Respiratory Tract Infections; Viruses.

\author{
Correspondencia a: \\ Diego F. García-Díaz \\ Departamento de Nutrición, \\ Facultad de Medicina, \\ Universidad de Chile. \\ Independencia 1027 \\ Santiago, Chile. \\ T: +56 229786759 \\ dgarcia@med.uchile.cl
}

U no de los aspectos que más afectan al diario vivir de cada individuo es el aumento del peso corporal ${ }^{1}$. A pesar de que el ser humano necesita de la presencia de tejido graso en su organismo, cuando este se desarrolla de forma excesiva se acarrean consecuencias muy perjudiciales para la salud ${ }^{2}$. La obesidad (incremento patológico en el organismo de las reservas energéticas en forma de grasa, acompañado de un aumento en la masa corporal total, debido al desequilibrio entre ingesta y gasto de energía ${ }^{3}$ ), se ha convertido en uno los problemas de salud más graves de las sociedades occidentales ${ }^{1}$. Inclusive, ha sido catalogada como la nueva epidemia del siglo XXI, debido principalmente a las enfermedades a las que este trastorno puede derivar ${ }^{1,2,4}$.
El exceso de energía disponible repercute directamente en la masa de células (adipocitos) del tejido adiposo blanco (TAB) que, debido a este fenómeno, aumentan su volumen tanto de forma hiperplásica (aumento de número) como hipertrófica (aumento de tamaño). Este engrosamiento (sobretodo el hipertrófico) de la masa del $\mathrm{TAB}$ es el que conduce a los diversos problemas clínicos relacionados con la obesidad, ya que el tejido se vuelve disfuncional, aumentando considerablemente la secreción de ácidos grasos libres al sistema, junto con la desregulación de la liberación de otras moléculas que actúan a nivel auto-, para- y endocrino ${ }^{5}$. El desajuste en la secreción de estas sustancias determinan el establecimiento de un estado pro-inflamatorio 
dañino para las células de este tejido, y que se ha descrito clave para el desarrollo de co-morbilidades $^{6}$. Esto implica que el TAB por sí mismo, y los productos lipídicos y proteicos derivados de este, ejercen una función importante tanto en la protección como en la susceptibilidad a desarrollar diversas anomalías.

Por otra parte, las infecciones respiratorias agudas (IRA) son una de las enfermedades infecciosas más frecuentes del ser humano, representando una importante causa de morbimortalidad en pediatría. Los niños pueden presentar entre seis y ocho infecciones respiratorias al año, siendo los virus los agentes infecciosos más frecuentes. Estas infecciones afectan a niños de todas las edades, si bien predominan por debajo de los cinco años y son especialmente prevalentes en menores de dos. En este último grupo de pacientes, estas infecciones suponen una de las causas más frecuentes de hospitalización a causa de infección respiratoria aguda baja (IRAB) originando numerosas consultas médicas tanto a nivel de atención primaria ambulatoria como de los servicios de urgencias hospitalarios ${ }^{7}$.

Entre los virus más comúnmente asociados con IRAB están los denominados "virus respiratorios clásicos": virus respiratorio sincicial (VRS), adenovirus, virus influenza A y B y virus parainfluenza $(\mathrm{VPI})^{8,9}$. Recientemente, la biología molecular y la utilización de sus herramientas en la detección de virus respiratorios permitió ampliar el número de estos patógenos. Entre ellos encontramos metapneumovirus humano (HMPV), rinovirus (RV) y enterovirus $^{10-13}$. Además, se identificaron nuevos agentes dentro de familias víricas conocidas, como los coronavirus (HCoV) HKU-1,10 NL-6311 y el agente responsable del síndrome de dificultad respiratoria aguda grave (SARS) HCoV-SARS, bocavirus $(\mathrm{HBoV})$ y el virus de la influenza $\mathrm{A}$ (H1N1) 2009, causante de la primera pandemia del siglo XXI ${ }^{14-18}$.

Es justamente en esta reciente pandemia de virus influenza $\mathrm{A}(\mathrm{H} 1 \mathrm{N1}$ ) 2009, donde la obesidad fue identificada como un nuevo factor de riesgo independiente para múltiples marcadores de severidad, incluyendo hospitalización, admisión en unidad de cuidados intensivos y muerte posterior a una infección ${ }^{19}$. Por lo tanto, el objetivo del presente trabajo fue revisar las evidencias científicas que existen que relacionan obesidad y gravedad de la infección respiratoria viral en niños.

\section{Material y Método}

Se llevó a cabo una búsqueda bibliográfica mediante el uso de la base de datos Pubmed. La estrategia de búsqueda utilizada comprendió los términos: [virus OR viral] AND respiratory AND children AND obesity. Esta búsqueda arrojó 40 resultados. De estos, se excluyeron aquellos artículos que no tenían relación directa con el tema de estudio (por ejemplo, relacionados con apnea, asma y otras condiciones alérgicas), y también donde no se informó número o porcentaje de pacientes pediátricos estudiados, y número o porcentaje de obesidad en la población estudiada. Tras esta revisión, se seleccionaron 9 artículos para el análisis. Por último, fueron incluidos artículos adicionales (38; seleccionados en base a relevancia y fecha de publicación) que fueron utilizados únicamente para poder sustentar lo observado en estos 9 artículos. La estrategia de revisión se grafica en la Figura 1.

\section{Resultados}

\section{Incidencia de obesidad en niños chilenos}

De acuerdo a lo reportado por la última encuesta de salud realizada por el gobierno, aproximadamente 1 de cada 4 chilenos presenta obesidad $^{20}$. Además, si comparamos las encuestas realizadas por el ministerio los años 2003 y 2010 se observa que la prevalencia de sobrepeso ha aumentado desde $37,8 \%$ a $39,3 \%$, y de obesidad desde $23,2 \%$ a $25,1 \%^{20,21}$. De acuerdo a estos datos, nos encontramos en claro aumento de la incidencia de esta patología. Este escenario negativo se comienza a manifestar debido a tendencias nutricionales tempranas durante la vida. Se ha descrito que niños que son obesos a los 6 años tienen $25 \%$ de probabilidad de ser obesos en la adultez, y para aquellos que lo son a los 12 años, el riesgo aumenta a $75 \% 22$. En Chile, la prevalencia de obesidad infantil se ha triplicado en el último tiempo; en escolares de primer año básico, ésta se incrementó desde $7 \%$ en 1987 a 18,5\% en $2005^{25}$. De hecho, el último reporte de la JUNAEB muestra que la prevalencia de obesidad en escolares de pre-kinder, kinder, $1^{\circ}$ básico y I medio es $22 \%$, $23 \%, 26, \%$ y $12 \%$, respectivamente ${ }^{23}$. Más aún, en el año 2009, de acuerdo a lo reportado por el MINSAL, 9,4\% de menores de 6 años presentaban 


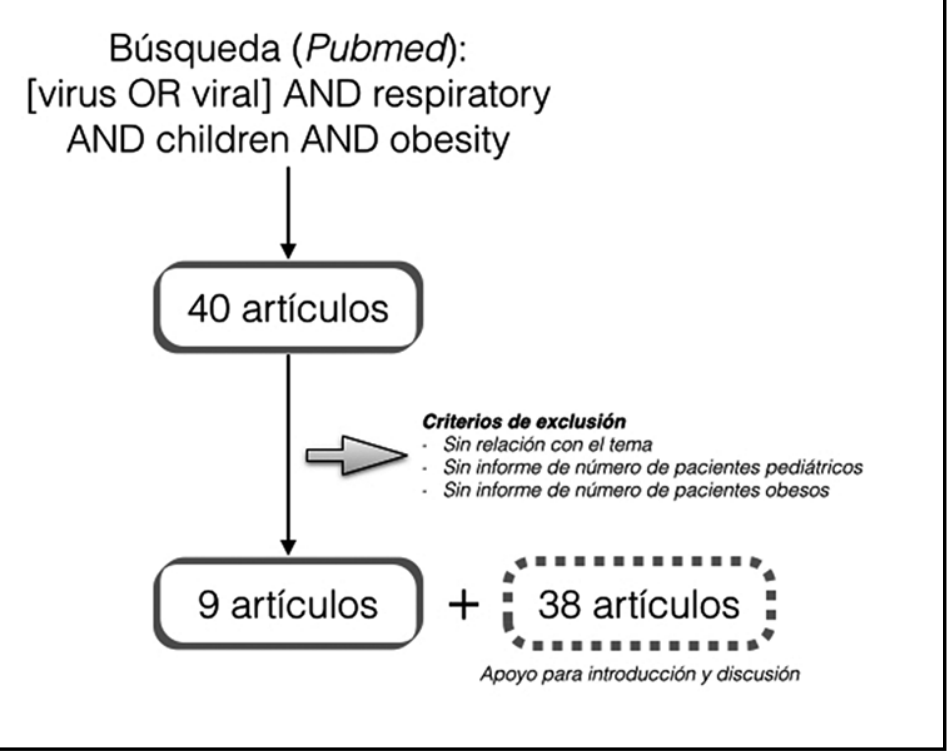

Figura 1. Estrategia de búsqueda.

obesidad, mientras que para el 2012 se reportó $10,3 \%$ de prevalencia ${ }^{24}$. En términos generales, la obesidad infantil se presenta actualmente en $6 \%$ en niños 2-3 años, $11 \%$ en niños de 3-4 y $16 \%$ de 4-5. En este sentido, Chile se hace merecedor de la sexta posición entre los países con mayor obesidad infanto-juvenil de la OCDE ${ }^{25}$.

Ahora bien, como ya se mencionó la obesidad que aparece temprano en el desarrollo y se consolida en la pubertad, presenta una alta probabilidad de mantenerse en edad adulta y relacionarse con la presencia de otras enfermedades crónicas no transmisibles. Por otro lado, obesidad infantil se relaciona, en la inmediatez, con baja autoestima, dificultades de socialización, bajo rendimiento escolar, incluyendo algunas otras consideraciones medicas aisladas de menor impacto. No obstante, en el presente trabajo se pretende revisar la evidencia cada vez más creciente respecto a otras complicaciones importantes que podrían impactar a niños que especialmente presentan obesidad.

\section{Enfermedad respiratoria en niños chilenos: incidencia y etiología}

En Chile, la IRAB constituye un importante problema de salud pública, siendo unas de las principales causas de hospitalización en pediatría. Según datos del MINSAL, los cuadros más frecuentes de IRAB corresponden a cuadros bronquiales obstructivos, que dan cuenta de $20 \%$ del total de las consultas respiratorias en Atención Primaria de Salud (APS) constituyendo la principal causa específica de morbilidad pediátrica en Chile ${ }^{7}$. Un estudio realizado por Lozano y cols. en el año $2008^{26}$, encontró la presencia de al menos un virus en $60 \%$ de los casos de niños hospitalizados por IRAB. Este trabajo demuestra la importancia de los virus en etiología de la IRAB en niños chilenos y el impacto que tiene la infección por VRS, siendo el principal agente causante de bronquiolitis (42-70\%) y de bronconeumonías en estos pacientes, concordante con lo encontrado por otros estudios ${ }^{27}$.

Durante el año 2014, según la Red de Vigilancia de Virus Respiratorios del Instituto de Salud Pública (ISP) ${ }^{28}$, de un total de 11.382 muestras positivas de IRA al mes de noviembre, predominó VRS (60\%), seguido de influenza A (14\%), parainfluenza (10\%), adenovirus (7\%), metaneumovirus $(6 \%)$ e influenza B (2\%). A diferencia de lo observado en el 2013 donde se encontró influenza $\mathrm{A}(\mathrm{H} 1 \mathrm{~N} 1) \mathrm{pdm} 09$, en el reciente pasado año los casos de influenza A corresponden principalmente a $\mathrm{A}(\mathrm{H} 3 \mathrm{~N} 2)$. De estos 11.382 casos de IRA, se notificaron 3.239 casos de IRAB en los hospitales centinelas con una positividad global de $46 \%$ para virus respiratorios ( 1.479 casos). Respecto al total 
de casos de IRAB positivos a virus respiratorios, predomina VRS (58\%), seguido de influenza $\mathrm{A}(\mathrm{H} 3 \mathrm{~N} 2)(17 \%)$, parainfluenza (10\%) y metaneumovirus (7\%), como los virus más frecuentes.

\section{Evidencia de la relación entre obesidady complicaciones en enfermedad respiratoria viral}

Yuy cols. ${ }^{29}$ estudiaron una serie de 1.657 niños chinos con confirmación de laboratorio de influenza A H1N1 de los cuales $42 \%$ presentó obesidad. Dentro de este grupo de pacientes obesos, se encontró que la proporción de niños obesos que presentaron complicaciones (ingreso a UCI o fallecimiento) fue significativamente mayor a los pacientes con influenza A H1N1 que no presentaron complicaciones (OR 1,40; IC 95\% 1,171,69; $\mathrm{p}<001)$. Por otra parte, Charland y cols. en un estudio realizado en pacientes menores de 18 años hospitalizados por infección respiratoria tipo influenza de 19 Estados de Estados Unidos de Norteamérica entre los años 2002 a 2008 (en período de invierno donde predominan los virus tipo influenza según lo informado por el Centro para el Control y Prevención de Enfermedades (CDC)), encontraron que un incremento en $5 \%$ en la prevalencia de obesidad fue asociada con $25 \%$ de incremento en tasas de hospitalización por enfermedades de este tipo (RR 1,25; IC 95\%1,18$1,32)^{30}$. Los casos reportados en este trabajo fueron incluidos sólo según los Códigos de Diagnóstico Internacionales (ICD), de los cuales se reconoce que son altamente específicos pero no sensibles, por lo que podría estar subvalorado el número de pacientes con hospitalización por enfermedad tipo influenza.

VRS sigue siendo el principal agente etiológico de infección respiratoria aguda baja en niños menores de 2 años. En el año 1999, Rivera-Claros y cols. ${ }^{31}$ concluyeron que en lactantes chilenos con IRAB causada por VRS, sin patología asociada, la prevalencia de sobrepeso y obesidad era alta (14\% y $8 \%$ respectivamente), mostrando una peor evolución clínica que los pacientes sin sobrepeso. Los lactantes obesos requirieron oxígeno por más tiempo comparado con el resto de los pacientes ( 5 días vs 3 días, $\mathrm{p}<0,05)$. Akiyama y cols. realizaron un estudio en 243 niños menores de 7 años donde se sugiere que la duración de la enfermedad por VRS (tanto días de sibilancias como días de fiebre) puede ser prolongada no sólo en niños con bajo peso sino que también en niños obesos ${ }^{32}$.
Por el contrario, en algunos estudios no se ha encontrado relación entre obesidad y gravedad de la infección respiratoria de origen viral en pacientes pediátricos. Un estudio realizado en la ciudad de Santa Fe, Argentina, por Kusznierz y cols. ${ }^{33}$ incluyó a 68 pacientes menores de 15 años hospitalizados por influenza pandémica. En este grupo de pacientes ninguno fue obeso. Otro estudio realizado en Argentina ${ }^{34}$, que recoge datos de pacientes fallecidos por influenza pandémica de 7 provincias, indica que en menores de 5 años, la obesidad no es un factor de riesgo frecuente. Sin embargo, en este estudio se agrupó a 179 pacientes en un rango de edad de 5 a 49 años, donde 35\% presentó obesidad, sugiriendo que sí puede ser un factor de riesgo de infección severa por influenza A H1N1. Un estudio realizado en España por Cardeñosa y cols. ${ }^{35}$ concluye que la obesidad no fue un factor de riesgo en población infantil con influenza A H1N1. No obstante, en este estudio sólo se consideró un grado elevado de obesidad (obesidad mórbida, IMC mayor o igual a 40) y no se describe si parte de los pacientes estudiados presentaron un menor grado de esta patología. Por otra parte, Lucas $^{36}$ publicó una revisión de la autopsia de 19 pacientes menores de 15 años que murieron por infección respiratoria por influenza A H1N1 en Inglaterra. Ninguno de estos niños presentaba obesidad y todas las muertes fueron asociadas a enfermedades congénitas. Además, en este mismo país, Mylesy cols. estudiaron una serie de 480 niños menores de 16 años con influenza pandémica, y sólo encontraron $0,6 \%$ de niños obe$\operatorname{sos}^{37}$. Los datos más importantes de los estudios analizados se resumen en la Tabla 1.

\section{Mecanismos posiblemente involucrados en la relación entre obesidad y enfermedad respiratoria viral}

Poco se sabe acerca de los mecanismos que sustentan el aumento de la susceptibilidad a consecuencias graves de pacientes obesos con, por ejemplo, influenza A H1N1. Por un lado, se sabe que la obesidad afecta negativamente la mecánica pulmonar. Sin embargo, pruebas recientes sugieren que el efecto inmunomodulador de la obesidad puede desempeñar un papel significativo en el resultado de una infección respiratoria viral. Previamente ya se ha documentado la relación entre nutrición y función respiratoria. Un estado nutricional deteriorado afecta negativamente la 
Tabla 1. Porcentaje de obesidad en niños con infección respiratoria de origen viral

\begin{tabular}{|lccc|}
\hline Referencia (año) & n pacientes & Edad (años) & Pacientes obesos (\%) \\
\hline Rivera-claros et al (1999) & 130 & $0-2$ & 8 \\
\hline Yu et al (2011) & 1.657 & $2-17$ & 42 \\
\hline Akiyama et al (2011) & 243 & $0-6$ & 30 a \\
\hline Cardeñosa et al (2011) & 168 & $0-14$ & $0^{\text {b }}$ \\
\hline Charland et al (2012) & $c$ & $0-18$ & 1,25 \\
\hline Kusnierz et al (2013) & 56 & $0-14$ & 0 \\
\hline Myles et al (2012) & 480 & $0-15$ & 0,6 \\
\hline Lucas (2010) & $19^{d}$ & $0-15$ & 0 \\
\hline Balanzat et al (2012) & 40 & $0-4$ & 0 \\
\hline
\end{tabular}

${ }^{a}$ Contabilizados desde la figura descrita en el artículo. Obesidad fue considerada según cálculo de tasa de obesidad descrita en el trabajo. 'bólo se considera pacientes con obesidad mórbida. 'Se infiere que es un número elevado ya que la muestra total es de 3.076.699 incluyendo adultos y niños de 19 estados de Estados Unidos de Norteamérica. 'Todos pacientes que fallecieron.

evolución de la enfermedad respiratoria, comprometiendo principalmente la estructura y función de músculos respiratorios, la capacidad ventilatoria y la función inmune ${ }^{38}$. Se ha descrito que la obesidad también es un factor de riesgo de morbilidad respiratoria, condicionando una reducción del volumen pulmonar total, hipertrofia del paladar blando y úvula, estenosis laringotraqueales y disminución de la expansión torácica, aumento de los depósitos de grasa que pueden llevar a trastornos respiratorios, y que en grados extremos ocasionan el síndrome de apnea del sueño ${ }^{39}$.

La inflamación descrita durante el desarrollo de obesidad parece jugar un papel fundamental en el nexo con un peor curso clínico de la enfermedad respiratoria. El sobrecrecimiento del TAB lleva a una desregulación en la producción de productos endógenos del tejido, los cuales a menudo presentan propiedades pro-inflamatorias, como por ejemplo TNF- $\alpha$, IL-6, resistina, leptina, óxido nítrico y MCP- $1^{40}$. Además, el crecimiento excesivo del TAB lleva a la inhibición en la secreción de productos anti-inflamatorios, como la adiponectina. Por lo tanto, es conocido que un aumento en la adiposidad corporal es habitualmente acompañado de un estado de inflamación crónica de bajo grado en el tejido adiposo. Aparentemente este estado puede modificar el estado de defensa del hospedero. La inflamación en obesidad conlleva un remodelamiento de la inmunidad adaptativa e innata: existe infiltración de células del sistema inmune innato dentro del TAB, específicamente macrófagos, que presentan un fenotipo activado $(\mathrm{M} 1)^{41}$, un cambio en la población de linfocitos dentro del tejido (de CD4 a CD8 ${ }^{42}$ y fuera de él. Por otro lado, se ha descrito que sujetos obesos presentan niveles disminuidos de linfocitos $\mathrm{T}$ $\mathrm{CD} 8+$ e incrementados o disminuidos de $\mathrm{T}$ CD4+ comparados con sujetos normopesos en la circulación ${ }^{43}$. Más aún, se ha descrito que animales genéticamente obesos presentan atrofia a nivel del timo, por lo tanto una disminución en las poblaciones de células $\mathrm{T}$ circulares y en el bazo $^{44}$, además de una reducción en la actividad NK y por tanto una disminución en la respuesta frente a la presentación de antígenos, lo que puede repercutir en la respuesta a la vacunación ${ }^{45}$. También, se ha descrito que la exposición crónica a proteínas pro-inflamatorias derivadas de $\mathrm{TAB}$ puede derivar en una desensibilización de células inmunes a estímulos pro-inflamatorios durante una infección ${ }^{46}$. Estas observaciones sugieren que la obesidad puede resultar efectivamente en un menoscabo de la defensa del hospedero, relacionando este hecho con un aumento del riesgo de contraer infecciones ${ }^{47}$.

\section{Conclusión}

De acuerdo a la literatura revisada en el presente trabajo, se observa que existe relación en algunas experiencias entre estado nutricional con gravedad de la enfermedad respiratoria en niños. Estas evidencias se sustentarían sobre la base de 
que la inflamación presente en obesidad pareciese jugar un papel fundamental en el nexo con un peor curso clínico de la enfermedad respiratoria. A pesar de que existen evidencias donde no se observa relación entre inflamación/obesidad y curso de infección respiratoria, las investigaciones donde sí se han reportado asociaciones han involucrado un número importante de casos.

Chile presenta una de las tasas más altas de obesidad infantil dentro de los países de la OCDE, tendencia que ha ido en aumento desde hace al menos 10 años. Debido a la tendencia en aumento de obesidad tanto a nivel nacional como mundial, y a la posibilidad de futuras nuevas pandemias de virus respiratorios, es necesario realizar más estudios que establezcan la relación entre obesidad y gravedad de la infección respiratoria viral, además de esfuerzos en educación nutricional. Particularmente en Chile, debido al elevado índice de obesidad en menores de 6 años y la posible relación entre obesidad y complicaciones en el curso de la IRA, las cuales son más frecuentes y graves en este período de vida, se hace indispensable establecer la relación entre ambas patologías. Para esto, se requiere el desarrollo de estudios de casos y controles y de cohorte en donde se pueda comparar el perfil de citoquinas y adipoquinas entre grupos de pacientes obesos y no obesos y relacionarlo con severidad clínica, eliminando el efecto de variables confundentes. En cuanto a la posibilidad de establecer medidas de control, sería importante poder establecer si existen diferencias entre niños obesos con lactancia materna exclusiva y con lactancia artificial, que en caso de presentar respuestas diferentes, se podría sugerir medidas para aminorar las posibles complicaciones durante la infección por VRS. Además, estos estudios servirían para concientizar a los padres con otra razón más por la cual es necesaria una buena alimentación para la mantención de un correcto estado nutricional. Por otra parte, como medida a corto plazo sería de interés proponer la incorporación en Guías Clínicas a la obesidad infantil como factor de riesgo frente a infecciones respiratorias para un mejor manejo de estos pacientes.

\section{Referencias}

1. Powers KA, Rehrig ST, Jones DB. Financial impact of obesity and bariatric surgery. Med Clin N Am 2007; 91 (3): 321-38.
2. Bray GA. Medical consequences of obesity. Journal of Clinical Endocrinology and Metabolism 2004; 89 (6): 2583-9.

3. Martínez JA. Body-weight regulation: causes of obesity. Proc Nutr Soc 2000; 59 (3): 337-45.

4. Sorensen TI, Virtue S, Vidal-Puig A. Obesity as a clinical and public health problem: is there a need for a new definition based on lipotoxicity effects? Biochim Biophys Acta 2010; 1801 (3): 400-4.

5. Iyer A, Fairlie DP, Prins JB, Hammock BD, Brown L. Inflammatory lipid mediators in adipocyte function and obesity. Nat Rev Endocrinol 2010; 6 (2): 71-82.

6. Pi-Sunyer X. The medical risks of obesity. Postgrad Med 2009; 121 (6): 21-33.

7. Ministerio de Salud. Departamento de Estadisticas e Informacion de Salud. Disponible en http://www.deis. cl [Consultado el 10 de septiembre de 2015].

8. Nokso-Koivisto J, Pitkaranta A, Blomqvist S, Jokinen J, Kleemola M, Takala A, et al. Viral etiology of frequently recurring respiratory tract infections in children. Clin Infect Dis 2002; 35 (5): 540-6.

9. García García ML, Ordobas Gabin M, Calvo Reya C, González Alvarez M, Aguilar Ruiz J, Arregui Sierra A, et al. [Viral infection of the lower respiratory tract in hospitalized infants: etiology, clinical features and risk factors]. An Esp Pediatr 2001; 55 (2): 101-7.

10. van den Hoogen BG, de Jong JC, Groen J, Kuiken T, de Groot R, Fouchier RA, et al. A newly discovered human pneumovirus isolated from young children with respiratory tract disease. Nat Med 2001; 7 (6): 719-24.

11. Renwick N, Schweiger B, Kapoor V, Liu Z, Villari J, Bullmann R, et al. A recently identified rhinovirus genotype is associated with severe respiratory-tract infection in children in Germany. J Infect Dis 2007; 196 (12): 1754-60.

12. McErlean P, Shackelton LA, Lambert SB, Nissen MD, Sloots TP, Mackay IM. Characterisation of a newly identified human rhinovirus, HRV-QPM, discovered in infants with bronchiolitis. J Clin Virol 2007; 39 (2): 67-75.

13. Jartti $T$, Lehtinen $P$, Vuorinen $T$, Osterback $R$, van den Hoogen B, Osterhaus AD, et al. Respiratory picornaviruses and respiratory syncytial virus as causative agents of acute expiratory wheezing in children. Emerg Infect Dis 2004; 10 (6): 1095-101.

14. Peiris JS, Lai ST, Poon LL, Guan Y, Yam LY, Lim W, et al. Coronavirus as a possible cause of severe acute respiratory syndrome. Lancet 2003; 361 (9366): 131925.

15. Drosten C, Gunther S, Preiser W, van der Werf S, Brodt HR, Becker S, et al. Identification of a novel coronavirus 
in patients with severe acute respiratory syndrome. $\mathrm{N}$ Engl J Med 2003; 348 (20): 1967-76.

16. Ksiazek TG, Erdman D, Goldsmith CS, Zaki SR, Peret T, Emery S, et al. A novel coronavirus associated with severe acute respiratory syndrome. N Engl J Med 2003; 348 (20): 1953-66.

17. Allander T, Tammi MT, Eriksson M, Bjerkner A, Tiveljung-Lindell A, Andersson B. Cloning of a human parvovirus by molecular screening of respiratory tract samples. Proc Natl Acad Sci USA 2005; 102 (36): 128916.

18. Hamilton $\mathrm{K}$. The emergence of the pandemic A/H1N1 2009 virus and its characteristics. Bull Mem Acad R Med Belg 2009; 164 (10): 260-3.

19. Centers for Disease, $C$ and Prevention. Intensive-care patients with severe novel influenza A (H1N1) virus infection-Michigan, June 2009. MMWR Morb Mortal Wkly Rep 2009; 58 (27): 749-52.

20. Ministerio de Salud. Encuesta Nacional de Salud 2010. Disponible en http://epi.minsal.cl/estudios-y-encuestas-poblacionales/encuestas-poblacionales/encuesta-nacional-de-salud/resultados-ens/ [Consultado el 11 de septiembre de 2015].

21. Ministerio de Salud. Encuesta Nacional de Salud 2003. Disponible en http://epi.minsal.cl/epi/html/invest/ENS/ InformeFinalENS.pdf [Consultado el 11 de septiembre de 2015].

22. Hodgson M. Obesidad infantil. Manual de Pediatria PUC. Disponible en http://escuela.med.puc.cl/paginas/ publicaciones/manualped/Presentac.html [Consultado el 10 de septiembre de 2015].

23. JUNAEB. Mapa Nutricional de la Junta Nacional de Auxilio Escolar y Becas 2013. Disponible en http://www. junaeb.cl/wp-content/uploads/2013/03/Informe-Mapa-Nutricional-2013.pdf [Consultado el 8 de enero de 2014].

24. Vio F. El preocupante incremento de la obesidad infantil en chile. 2013. Disponible en http://www.inta.cl/index. php?option $=$ com_content\&view $=$ article\&id $=502$ :obesidad-infantil\&catid=53:opinion\&Itemid $=134$ [Consultado el 10 de septiembre de 2015].

25. Ministerio de Salud. Panorama de Salud 2013 Informe OECD sobre Chile y comparación con países miembros. Disponible en http://web.minsal.cl/sites/default/files/ INFORME OCDE_2013_21 11_final.pdf [Consultado el 10 de septiembre de 2015].

26. Lozano J, Yáñez L, Lapadula M, Lafourcade M, Burgos F, Budnik I, et al. Lower respiratory tract infections in children: A prospective etiological study. Rev Chil Enf Respir 2008; 24 107-12.

27. Martínez P, Cordero J, Valverde C, Unanue N, Dalmaz- zo R, Piemonte P, et al. [Viral respiratory co-infections in pediatric patients admitted for acute respiratory infection and their impact on clinical severity]. Rev Chilena Infectol 2012; 29 (2): 169-74.

28. Instituto de Salud Pública. Vigilancia de virus respiratorios. Disponible en http://www.ispch.cl/virusrespiratorios [Consultado el 10 de septiembre de 2015].

29. Yu H, Feng Z, Uyeki TM, Liao Q, Zhou L, Feng L, et al. Risk factors for severe illness with 2009 pandemic influenza A (H1N1) virus infection in China. Clin Infect Dis 2011; 52 (4): 457-65.

30. Charland KM, Buckeridge DL, Hoen AG, Berry JG, Elixhauser A, Melton F, et al. Relationship between community prevalence of obesity and associated behavioral factors and community rates of influenza-related hospitalizations in the United States. Influenza Other Respir Viruses 2013; 7 (5): 718-28.

31. Rivera Claros R, Marín V, Castillo-Durán C, Jara L, Guardia S, Díaz N. [Nutritional status and clinical evolution of hospitalized Chilean infants with infection by respiratory syncytial virus (RSV)]. Arch Latinoam Nutr 1999; 49 (4): 326-32.

32. Akiyama N, Segawa T, Ida H, Mezawa H, Noya M, Tamez $\mathrm{S}$, et al. Bimodal effects of obesity ratio on disease duration of respiratory syncytial virus infection in children. Allergol Int 2011; 60 (3): 305-8.

33. Kusznierz G, Uboldi A, Sosa G, Torales S, Colombo J, Moyano C, et al. Clinical features of the hospitalized patients with 2009 pandemic influenza A (H1N1) in Santa $\mathrm{Fe}$, Argentina. Influenza Other Respir Viruses 2013; 7 (3): 410-7.

34. Balanzat AM, Hertlein C, Apezteguia C, Bonvehi P, Camera L, Gentile A, et al. An analysis of 332 fatalities infected with pandemic 2009 influenza A (H1N1) in Argentina. PLoS ONE 2012; 7 (4): e33670.

35. Cardenosa N, Rodes A, Follia N, Plasencia E, Lafuente $\mathrm{S}$, Arias $\mathrm{C}$, et al. Epidemiological analysis of severe hospitalized 2009 pandemic influenza A (H1N1) cases in Catalonia, Spain. Hum Vaccin 2011; 7 Suppl 226-9.

36. Lucas S. Predictive clinicopathological features derived from systematic autopsy examination of patients who died with A/H1N1 influenza infection in the UK 200910 pandemic. Health Technol Assess 2010; 14 (55): 83 114.

37. Myles PR, Semple MG, Lim WS, Openshaw PJ, Gadd EM, Read RC, et al. Predictors of clinical outcome in a national hospitalised cohort across both waves of the influenza A/H1N1 pandemic 2009-2010 in the UK. Thorax 2012; 67 (8): 709-17.

38. DeMeo MT, Van de Graaff W, Gottlieb K, Sobotka P, Mobarhan S. Nutrition in acute pulmonary disease. 
Nutr Rev 1992; 50 (11): 320-8.

39. Pi-Sunyer FX. Medical hazards of obesity. Ann Intern Med 1993; 119 (7 Pt 2): 655-60.

40. Ferrante AW. Obesity-induced inflammation: a metabolic dialogue in the language of inflammation. J Intern Med 2007; 262 (4): 408-14.

41. Hotamisligil GS. Molecular mechanisms of insulin resistance and the role of the adipocyte. Int J Obes Relat Metab Disord 2000; 24 Suppl 4 S23-7.

42. Kintscher U, Hartge M, Hess K, Foryst-Ludwig A, Clemenz M, Wabitsch M, et al. T-lymphocyte infiltration in visceral adipose tissue: a primary event in adipose tissue inflammation and the development of obesity-mediated insulin resistance. Arterioscler Thromb Vasc Biol 2008; 28 (7): 1304-10.

43. O’Rourke RW, Kay T, Scholz MH, Diggs B, Jobe BA, Lewinsohn DM, et al. Alterations in T-cell subset fre- quency in peripheral blood in obesity. Obes Surg 2005; 15 (10): 1463-8.

44. Karlsson EA, Sheridan PA, Beck MA. Diet-induced obesity impairs the $\mathrm{T}$ cell memory response to influenza virus infection. J Immunol 2010; 184 (6): 3127-33.

45. Talbot HK, Coleman LA, Crimin K, Zhu Y, Rock MT, Meece J, et al. Association between obesity and vulnerability and serologic response to influenza vaccination in older adults. Vaccine 2012; 30 (26): 3937-43.

46. Ziegler-Heitbrock HW, Wedel A, Schraut W, Strobel M, Wendelgass P, Sternsdorf T, et al. Tolerance to lipopolysaccharide involves mobilization of nuclear factor kappa B with predominance of p50 homodimers. J Biol Chem 1994; 269 (25): 17001-4.

47. Falagas ME, Kompoti M. Obesity and infection. Lancet Infect Dis 2006; 6 (7): 438-46. 